\title{
OPEN The C. elegans miR-235 regulates the toxicity of graphene oxide via targeting the nuclear hormone receptor DAF-12 in the intestine
}

\author{
Tiantian Guo ${ }^{1}$, Lu Cheng ${ }^{1}$, Huimin Zhao ${ }^{1}$, Yingying Liu ${ }^{1}$, Yunhan Yang ${ }^{1}$, Jie Liu² \& Qiuli Wu ${ }^{1} \bowtie$
}

The increased application of graphene oxide (GO), a new carbon-based engineered nanomaterial, has generated a potential toxicity in humans and the environment. Previous studies have identified some dysregulated microRNAs (miRNAs), such as up-regulated mir-235, in organisms exposed to GO. However, the detailed mechanisms of the dysregulation of miRNA underlying GO toxicity are still largely elusive. In this study, we employed Caenorhabditis elegans as an in vivo model to investigate the biological function and molecular basis of mir-235 in the regulation of GO toxicity. After low concentration GO exposure, mir-235 (n4504) mutant nematodes were sensitive to GO toxicity, implying that mir-235 mediates a protection mechanism against $\mathrm{GO}$ toxicity. Tissue-specific assays suggested that mir-235 expressed in intestine is required for suppressing the GO toxicity in C. elegans. daf-12, a gene encoding a member of the steroid hormone receptor superfamily, acts as a target gene of mir-235 in the nematode intestine in response to GO treatment, and RNAi knockdown of daf-12 suppressed the sensitivity of mir-235(n4503) to GO toxicity. Further genetic analysis showed that DAF-12 acted in the upstream of DAF-16 in insulin/IGF-1 signaling pathway and PMK-1 in P38 MAPK signaling pathway in parallel to regulate GO toxicity. Altogether, our results revealed that mir-235 may activate a protective mechanism against $G O$ toxicity by suppressing the DAF-12-DAF-16 and DAF-12PMK-1 signaling cascade in nematodes, which provides an important molecular basis for the in vivo toxicity of $\mathrm{GO}$ at the miRNA level.

Graphene oxide (GO), a novel engineering nanomaterial composed of carbon atoms with surface-rich functional groups, has been widely used in various areas owing to its excellent physicochemical characteristics ${ }^{1-5}$. Meanwhile, effect of GO exposure to human and environmental animals has aroused extensive attention with its application. It has been reported that $\mathrm{GO}$ exposure can induce toxicity including high level of reactive oxygen species (ROS), cell apoptosis and inflammatory in vitro and in vivo ${ }^{6-9}$. However, the molecular mechanisms of organisms response to GO toxicity are largely unknown.

Caenorhabditis elegans (C. elegans) is a powerful model animal for genetic investigation of various biological processes ${ }^{10}$. Owing to its sensitivity to environmental toxicants, C. elegans has also been widely used as an in vivo model for studying the toxicity assessment and toxicological mechanisms of environmental toxicants ${ }^{11}$. Previous studies have found that GO exposure causes a short lifespan, attenuate athletic ability, reduced reproductive capacity and increased intestinal ROS in C. elegans ${ }^{7}$. Meanwhile, several important molecular signaling pathways including c-Jun N-terminal kinase (JNK), p38 MAP Kinase (MAPK), insulin growth factor-1 (IGF-1), transforming growth factor- $\beta(\mathrm{TGF} / \beta)$, Wnt, oxidative stress associated, apoptosis, and DNA damage signaling pathways have been identified to be involved in regulating GO toxicity in nematodes ${ }^{12-17}$.

Recent studies have indicated that some microRNAs (miRNAs) may function in the control of GO toxicity, which further improves our understanding of the molecular mechanism of GO toxicity ${ }^{18-20}$. miRNAs are a class of endogenous nucleotide non-protein-encoding RNAs with 21-23 bases, and regulate eukaryotic gene expression at the post-transcriptional level ${ }^{21,22}$. miRNAs inhibit gene expression primarily by binding to certain sites of the 3' untranslated regions ( $3^{\prime}$ UTRs) of target mRNAs, which results in degradation of mRNA and inhibition of protein translation ${ }^{23-25}$. In C. elegans, miRNAs also play important roles in response to the toxicity of certain carbon nanomaterials. For example, let-7, mir-259, mir-35, and mir-355 are involved in regulating the

${ }^{1}$ Institute of Nephrology, Zhong Da Hospital, Medical School, Southeast University, Nanjing, China. ${ }^{2}$ Monash Biomedicine Discovery Institute and Department of Anatomy and Developmental Biology, Monash University, Melbourne, VIC 3800, Australia. ${ }^{\circledR}$ email: qlwu@seu.edu.cn 
multi-walled carbon nanotubes (MWCNTs) toxicity in C. elegans ${ }^{26-29}$. Moreover, let-7 acted as a downstream target for epidermal BLI-1 in the regulation of GO-PEG toxicity ${ }^{20}$.

In previous studies, we have found several dysregulated miRNAs in GO exposed nematodes, and tested the function of candidate miRNAs in regulating GO toxicity ${ }^{30}$. Among these dysregulated miRNAs, mir-235 was upregulated in GO-exposed nematodes. mir-235, a sole orthologue of mammalian miR-92 in oncogenic miR-17-92 cluster, acts in the hypodermis and glial cells to arrest postembryonic developmental events in neuroblasts and mesoblasts ${ }^{31}$. mir-235 is induced by dietary restriction at the end of larval development, which subsequently suppresses Wnt signaling by inhibiting cwn-1/WNT4 and thereby promotes longevity ${ }^{32}$. Our previous study found that mir-235(n4504) mutants display a sensitive property to GO toxicity ${ }^{30}$. However, it is still unclear about the molecular basis of mir-235 in response to the GO toxicity at low concentration.

In this study, we investigated the molecular mechanisms of mir-235 in regulating GO toxicity using the in vivo assay system of $C$. elegans. We found that mir-235 regulates GO toxicity via targeting daf-12 in the intestine. The nuclear hormone receptor DAF-12, a homolog of vertebrate vitamin D and liver X receptors, functions as a ligand-dependent switch that regulates the developmental progression and arrest in response to environmental cues $^{33-35}$. Our results indicated that the intestinal mir-235/DAF-12 acted the upstream of both DAF-16 in the insulin/IGF-1 and PMK-1 in p38 MAPK signaling pathway in parallel to regulate GO toxicity in nematodes.

\section{Results}

Physicochemical properties of prepared GO. The ultrasound-treated GO was a single layer of nanosheet, the thickness of which was approximately $1.0 \mathrm{~nm}$ based on AFM assays (Fig. S1A). After sonication, the size distribution of most GO was in the range of 40-50 nm (Fig. S1B). Raman spectroscopy assay showed that GO had a D band $\left(1354.99 \mathrm{~cm}^{-1}\right.$ ) and a G band $\left(1599.04 \mathrm{~cm}^{-1}\right)$, respectively (Fig. S1C). The D band reflected the disorder of graphite layer introduced after treatment with sulfuric acid and $\mathrm{KMnO}_{4}$. Zeta potential of GO in $\mathrm{K}$ medium was $-22.3 \pm 2.5 \mathrm{mV}$.

mir-235 acts in the intestine to control GO toxicity. Our previous studies have suggested that the mutation of mir-235 induced a sensitive property to GO concentration of $10 \mathrm{mg} / \mathrm{L}$ in C. elegans ${ }^{30}$. However, most nanomaterials released into the environment are in the range of $\mathrm{ng} / \mathrm{L}$ or $\mu \mathrm{g} / \mathrm{L}^{36,37}$. To further determine the function of mir-235 in response to GO toxicity at the low-concentration, we quantified the ROS production and locomotion behavior of mir-235(n4504) mutant nematodes exposed to $100 \mu \mathrm{g} / \mathrm{L} \mathrm{GO}$. After the prolonged exposure from L1-larvae for $96 \mathrm{~h}$, we found that mir-235 mutant nematodes generate more ROS and display a decreased locomotive speed, compared with control animals, which suggests that mir-235 mutant nematodes still sensitive to GO exposure (Fig. S2).

As mir-235 is expressed in intestine, epidermis, and neurons ${ }^{31}$, we used the tissue-specific promoter to investigate the tissue-specific activity of mir-235 in the regulation of GO toxicity. Rescue assay by expression of mir-235 in the neurons or epidermis with the $u n c-14$ or $d p y-7$ promoter did not significantly affect the sensitive property of mir-235(n4504) mutant to GO toxicity (Fig. 1). In contrast, the expression of mir-235 under the control of the intestine-specific ges-1 promoter significantly decreased the intestinal ROS production and increased locomotion behavior in mir-235(n4504) mutant exposed to GO (Fig. 1). These results indicated that mir-235 may act in the intestine to mediate a protection mechanism against GO toxicity in nematodes.

Intestinal candidate targeted genes of mir-235 after GO exposure. To identify molecular targets of mir-235 in the regulation of GO toxicity, we predicted the possible targeted genes of mir-235 by TargetScan database. In view of mir-235 function in the intestine to control GO toxicity, 50 target genes expressed in the intestine were further screened out from 194 possible targeted genes of mir-235 (Table S1).

To identify the mir-235 targeted genes in C. elegans intestine, we isolated the intestine and extracted the RNA as previously reported ${ }^{39}$. Our genetic assays of 50 predicted genes showed that the expression levels of C52B9.4, mel-11, C34D4.4, T28D9.1, ifc-2, daf-12 and nhr-71 were decreased, and the expression levels of aex-3, soap-1, F27D9.2 and C42C1.4 were increased in wild-type N2 intestine after GO exposure (Fig. 2A). Considering the increased expression of mir-235 in wild-type N2 exposed to GO, we further focus on these genes with decreased expression after GO exposure in the intestine of wild-type N2. We found that the expression levels of mel-11, T28D9.1 and daf-12 were increased in the intestine of mir-235(n4504) mutant compared with that in the intestine of wild-type N2 (Fig. 2B). Therefore, the results suggest that these 3 genes (mel-11, T28D9.1 and daf-12) may be candidate targets for mir-235 in the intestine.

Intestinal daf-12 is a potential mir-235 target in the regulation of GO toxicity. daf-12 encodes a member of the steroid hormone receptor superfamily homologous to human vitamin D receptor ${ }^{33-35}$. Among the 3 candidate target genes analyzed above, the expression level of $d a f-12$ was increased most significantly in mir-235( $n 4504$ ) exposed to GO, which implied that daf-12 plays a key role in response to GO toxicity (Fig. 2B). Therefore, we analyzed the effect of GO exposure on the intestinal ROS production and locomotion endpoints of daf-12(rh61rh411) and daf-12(sa204) mutants. After GO exposure, we found that daf-12(rh61rh411) and daf-12(sa204) mutants caused the resistance of nematodes to GO toxicity in inducing ROS production and in decreasing locomotion behavior (Fig. 3A,B).

Because mir-235 acts in the intestine to regulate GO toxicity in nematodes and daf-12 gene is also expressed in the intestine ${ }^{38}$, we next focus on whether intestinal daf-12 directly respond to GO toxicity. Using intestine-specific interference nematodes (VP303), we found that intestinal-specific RNAi knockdown of daf-12 significantly inhibited GO toxicity in inducing intestinal ROS production (Fig. 3C). That is, daf-12 acts in the intestine to 
A
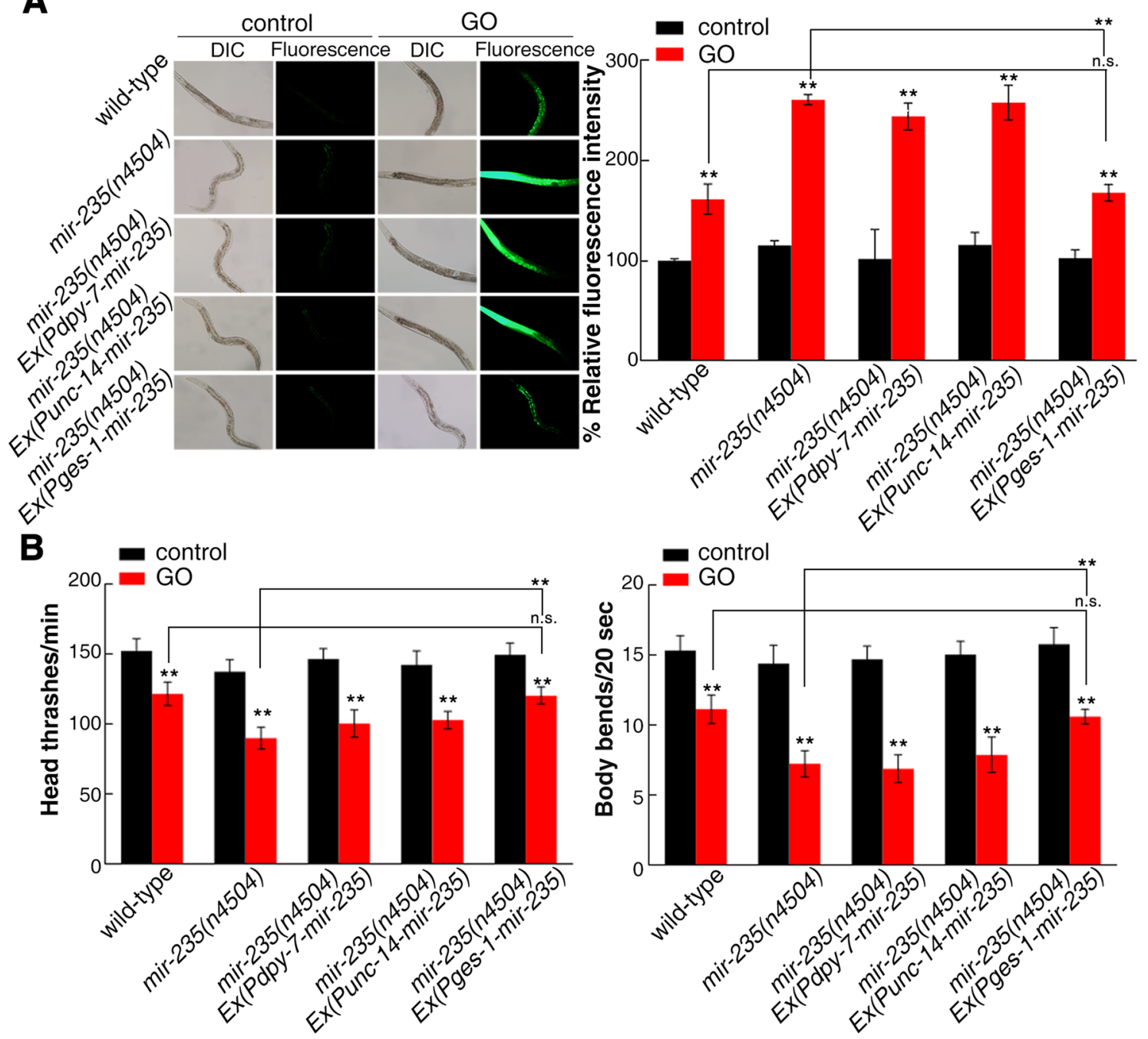

Figure 1. Tissue-specific activity of mir-235 in regulating GO toxicity. (A) Tissue-specific activity of mir-235 in regulating GO toxicity on ROS production. (B) Tissue-specific activity of mir-235 in regulating GO toxicity on locomotion behavior in nematodes. Bars represent means \pm SD. n.s. represents no significant difference. ${ }^{\star *} p<0.01$ versus control (if not specially indicated).

regulate GO toxicity in nematodes, which also implies that daf-12 is a potential target for mir-235 in regulating GO toxicity in the intestine of nematodes.

In vivo 3' UTR binding assay of daf-12 with mir-235. To further confirm whether daf-12 is a direct target of mir-235, we firstly predicted the binding site of daf-12 3' UTR with mir-235 by Targetscan. It suggested that daf-12 3' UTR had 7 bases complementary to mir-235 (Table S2 and Fig. 4A). We then constructed transgenic nematodes which contain a GFP vector driven by intestinal-specific ges-1 promoter and GFP reporter under the control of the daf-12 $3^{\prime}$ UTR (wild-type or mir-235 binding site mutated from GUGCAAU to GAACAAU) (Fig. 4A,B). Pges-1::mCherry unc-54 3' UTR was used as a control because mir-235 can not bind to unc-54 3' UTR. We found that the expression of daf-12 GFP in the intestine was significantly reduced after GO exposure in wild-type nematodes with daf-12 3' UTR (wild-type) (Fig. 4C). However, mutation of the putative binding site for mir-235 in daf-12 3' UTR abolished the reduction of GFP expression in wild-type nematodes (Fig. 4C) After GO exposure, the GFP expression of daf-12 3' UTR (wild-type) was significantly increased in mir-235(n4504) nematodes than that in wild-type nematodes (Fig. 4C). Thus, our analysis further supports that intestinal daf-12 is a direct targeted gene of mir-235 in regulating the response to GO toxicity.

Genetic interaction between mir-235 and daf-12 in regulating the response to GO toxicity. To further investigate the genetic interaction between mir-235 and daf-12 in the regulation of GO toxicity, we used daf-12 RNAi strain to interfere with mir-235(n4504) mutant to obtain nematodes mir-235(n4504); daf12(RNAi). After exposure to GO, mir-235(n4504); daf-12(RNAi) showed the similar phenotype with daf-12 RNAi knockdown nematodes in decreasing ROS production and increasing locomotion behavior (Fig. 5), which 


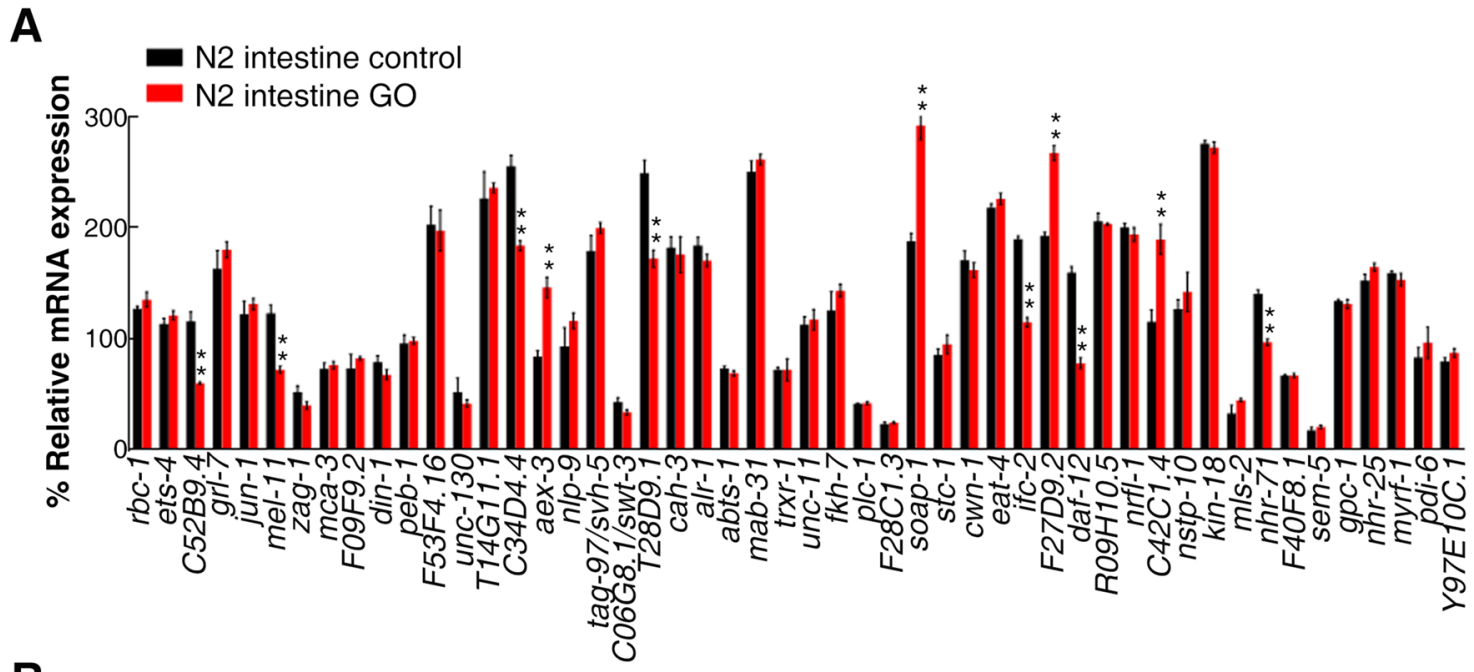

B

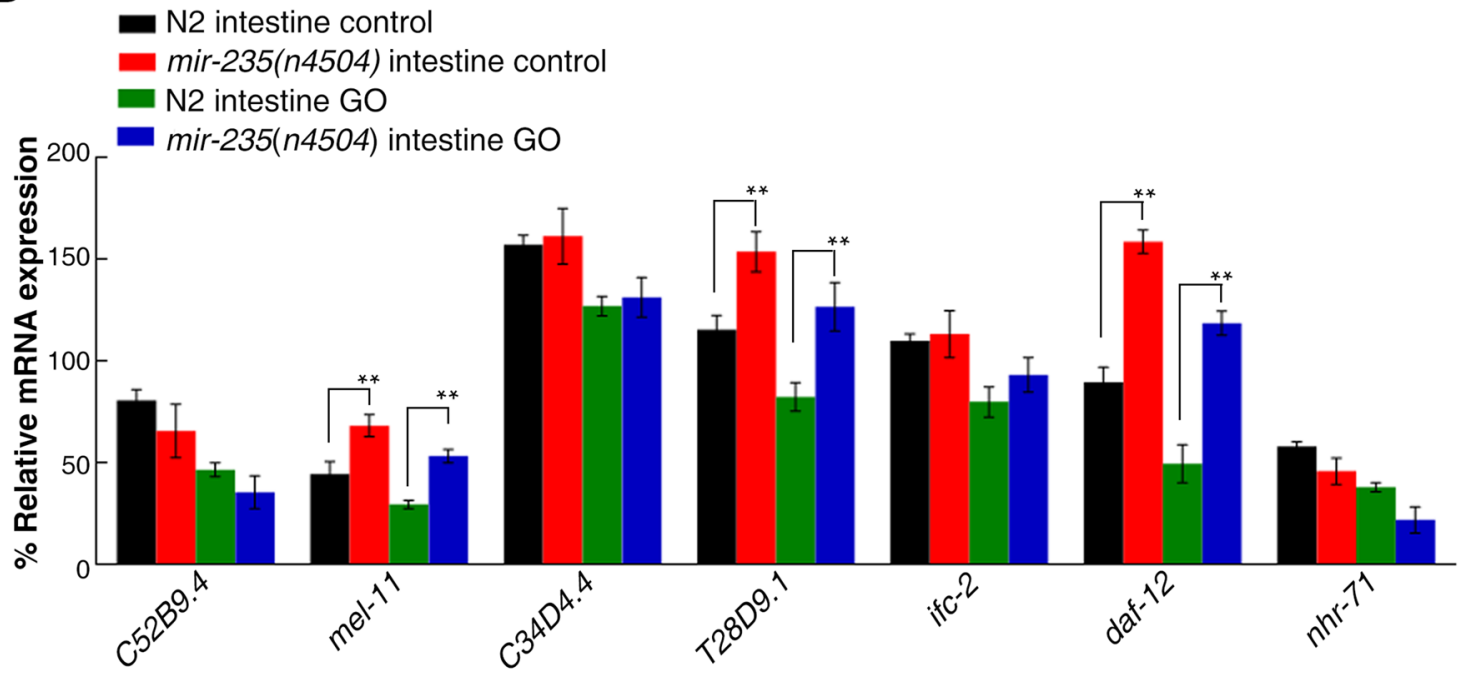

Figure 2. Validation of candidate intestinal targets of mir-235 in GO exposure nematodes via qRT-PCR analysis. (A) Dysregulated candidate intestinal targets of mir-235 in intestinal tract of wild-type nematodes exposed to GO. (B) Dysregulated candidate intestinal targets of mir-235 in intestinal tract of mir- 235 mutant nematodes exposed to GO. Bars represent means \pm SD. ${ }^{* *} p<0.01$. Triplicate independent experiments were performed for the assays.

indicated that daf-12 RNAi knockdown suppressed the susceptibility of mir-235 mutant nematodes. Therefore, daf-12 serves as a downstream gene of mir-235, and regulates GO toxicity by inhibiting the function of mir-235.

Effect of mir-235 and daf-12 on the distribution of GO in nematodes. Distribution and translocation of GO are key factors of the toxicity formation in vivo ${ }^{40}$. Previous study has showed that the fluorescent molecular probe Rho B can interact with GO because of its $\pi-\pi$ stacking ${ }^{41}$. By using UV-Vis to monitor the residual amount of Rho B in the solution after the loading process, it was found that almost $95 \%$ of the Rho B was loaded on the GO film ${ }^{41}$. At present, GO/Rho B has been used to reflect the distribution and translocation of $\mathrm{GO}$ in cells and in organisms ${ }^{40,42}$. Therefore, we used Rho B to label GO, which allows us to visualize the distribution of GO in nematodes. After GO/Rho B exposure, we found that only a small amount of GO was transported into the intestine in wild-type animals. In sharp contrast, mir-235(n4504) mutant nematodes have more GO in intestine (Fig. 6). Interestingly, RNAi knockdown of daf-12 in either wild type or mir-235(n4504) significantly reduced the intestinal distribution and translocation of GO (Fig. 6). These data indicated that the mir-235 mutation significantly enhanced the distribution and translocation of GO in nematodes, and RNAi knockdown of daf-12 suppressed distribution and translocation of GO in mir-235(n4504) mutant nematodes.

mir-235/DAF-12 regulated GO toxicity in the intestine by mediating the insulin/IGF-1 and p38 MAPK signaling pathways. Previous study has indicated that insulin/IGF-1 and p38 MAPK signaling pathways in the intestine regulate GO toxicity in C. elegans ${ }^{13,15}$. daf-16 gene encodes the transcriptional factor DAF-16/FOXO in the insulin signaling pathway ${ }^{43}$. PMK-1, as an ortholog of human MAPK14, exhibits MAP kinase activity and transcription factor binding activity in p38 MAPK signaling pathways ${ }^{13}$. To investigate 
A
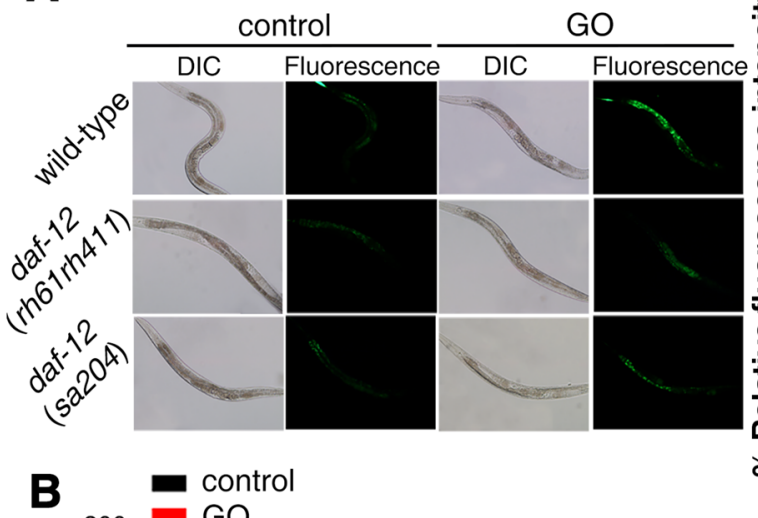
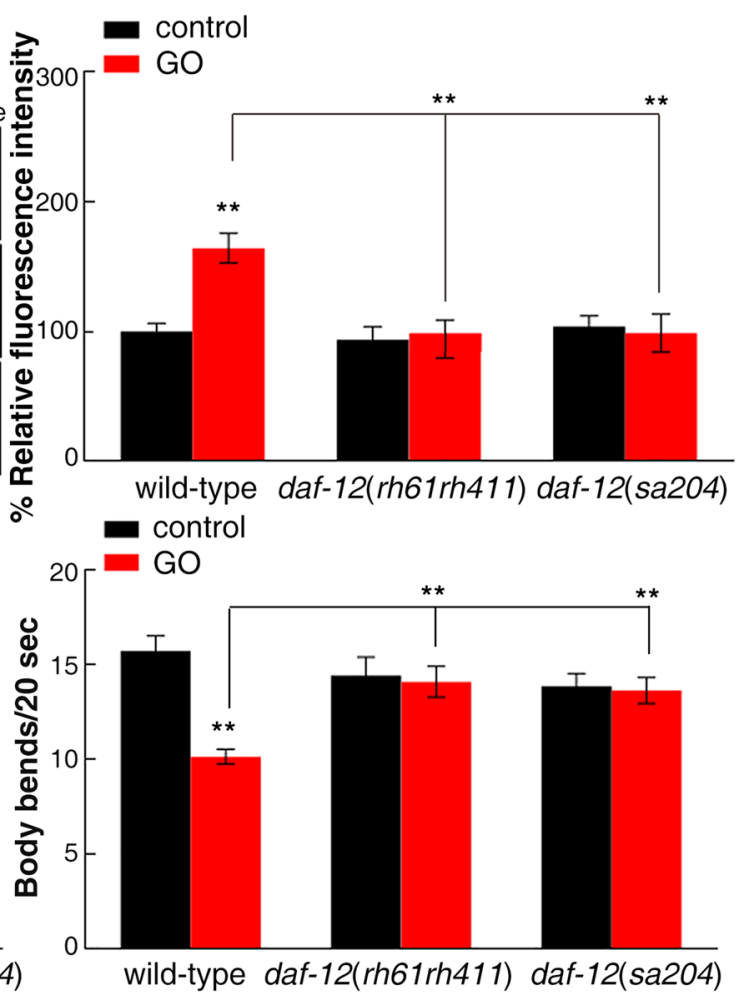
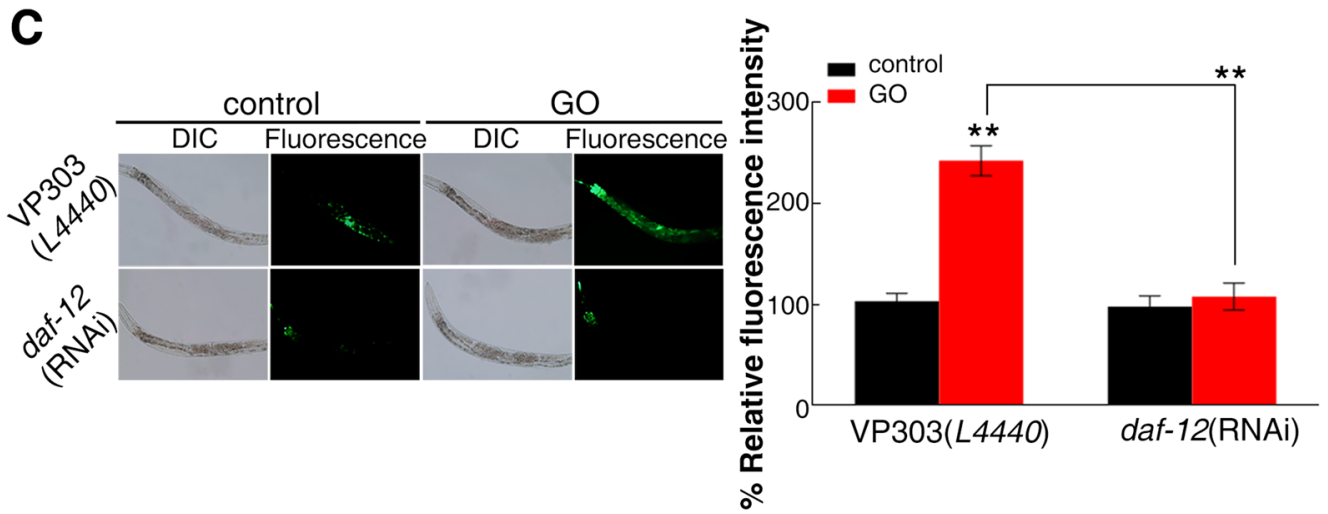

Figure 3. Effects of daf-12 mutation or intestine-specific RNAi knockdown on GO toxicity. (A) Effect of daf-12 mutation on GO toxicity in decreasing ROS production. (B) Effect of daf-12 mutation in regulating GO toxicity in increasing locomotion behavior. (C) Intestine-specific activity of daf- 12 in regulating GO toxicity in decreasing ROS production. Bars represent means \pm SD. ${ }^{* *} p<0.01$. Number of nematodes is $25-30$ per condition, and the experiment was repeated three times independently.

whether mir-235 regulates GO toxicity through insulin/IGF-1 and p38 MAPK signaling pathways, we explored the genetic interaction between mir-235 and daf-16 or pmk-1 in response to GO toxicity in nematodes. We observed that RNAi knockdown of daf-16 induced a sensitive property to GO toxicity in inducing ROS production and in decreasing locomotion behavior. Importantly, after GO exposure, the intestinal ROS production and the locomotion behavior in Ex(Pges-1-mir-235); daf-16(RNAi) nematodes were similar to those in daf-16 (RNAi) nematodes, which indicated that RNAi knockdown of daf-16 suppressed the resistance of mir-235 intestinal overexpression nematodes to the GO toxicity (Fig. 7). This result showed that mir-235 may act upstream of daf-16 to regulate GO toxicity in nematodes. Similarly, we also observed that RNAi knockdown of $p m k-1$ induced a sensitive property to GO toxicity and suppressed the resistance of mir-235 intestinal overexpression nematodes to the GO toxicity (Fig. 7). This result showed that mir-235 may also act upstream of $p m k-1$ to regulate GO toxicity in nematodes.

Considering intestinal daf-12 as a target of mir-235 to regulate GO toxicity, we further analyzed whether intestinal daf- 12 also regulated GO toxicity through insulin and p38 MAPK signaling pathway. After GO exposure, intestinal-specific RNAi knockdown of daf-12 significantly increased the daf-16 and pmk-1 expression based on the qRT-PCR results (Fig. 8A). In contrast, there was no significant change in the expression of daf-16 and pmk-1 in intestinal-specific RNAi nematodes of $d a f-12$ without GO exposure (Fig. S3). Further, we analyzed the subcellular localization of the DAF-16::GFP fusion protein and found that RNAi of daf-12 not only increased the 
A

mir-235 3'...AGUCCGGCCCCUCUCACGUUAU...5' (1)

WT daf-12 3' UTR 5'...ACAAAUAAAAGUGUAGUGCAAUG...3' Mutated daf-12 3' UTR 5'...ACAAAUAAAAGUGUAGAACAAUG...3'
B

\begin{tabular}{|l|l|l|}
\hline ges-1 promoter & GFP & WT or mutated daf-12 3' UTR \\
\hline ges-1 promoter & mCherry & unc-54 3' UTR \\
\hline ges-1 promoter & mir-235 & \\
\hline
\end{tabular}
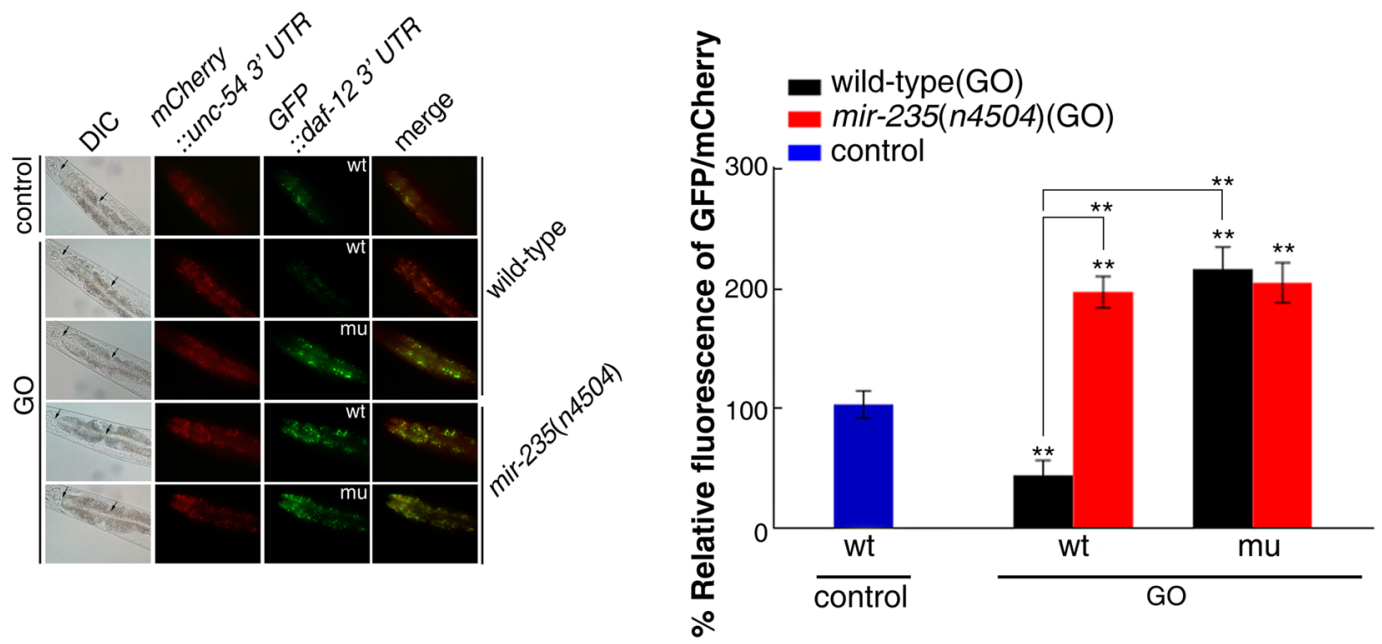

Figure 4. In vivo $3^{\prime}$ UTR binding assay of daf-12 with mir-235. (A) Predicted binding site on daf-12 3' UTR by Targetscan. Mutated nucleotides for experiments are indicated in red. WT, wild-type. (B) DNA construct outline. (C) Fluorescence images of the daf-12 3' UTR GFP reporter in nematodes exposed to GO. The fluorescence intensity of the first two pairs of intestinal cells was quantified (black arrows indicated quantitative area in DIC images). wt: wild-type. mu: mutation of the putative binding site for mir-235 in daf-12 $3^{\prime}$ UTR. Bars represent means \pm SD. ${ }^{* *} p<0.01$. Number of nematodes is $25-30$ per condition, and the experiment was repeated three times independently.

expression of DAF-16::GFP, but also enhanced the translocation of DAF-16::GFP into the nucleus of intestinal cells in GO exposed nematodes (Fig. S4).

Moreover, we knockdowned daf-12 with RNAi within daf-16(mu86) mutants to investigate the genetic interaction between daf-12 and daf-16 in the regulation of GO toxicity. The phenotype of daf-16(mu86); daf-12(RNAi) was similar to that of daf-16(mu86) mutants, based on the quantification of ROS production and locomotion behavior after GO exposure (Fig. 8B,C). Meanwhile, we also observed the phenotype of pmk-1(km25); daf12(RNAi) was similar to that of pmk-1(km25) mutants (Fig. 8B,C). These results suggest that daf-12 may play a role in regulating GO toxicity through acting upstream of $d a f-16$ or $p m k-1$.

To explain whether there was an interaction between $d a f-16$ and $p m k-1$ in regulating GO toxicity, we next constructed daf-16(RNAi); pmk-1(RNAi) nematodes. We observed that daf-16 and pmk-1 knockdown nematodes induced a susceptibility to GO toxicity in inducing intestinal ROS production and in decreasing locomotion behavior. By contrast, daf-16(RNAi); pmk-1(RNAi) nematodes were more susceptible to the GO toxicity than daf-16(RNAi) or pmk-1(RNAi) nematodes respectively (Fig. S5), indicating that two signaling pathways acted in parallel to regulate GO toxicity. Altogether, these findings implied that mir-235/DAF-12 may mediate the insulin/IGF-1 and p38 MAPK signaling pathways in parallel to regulate GO toxicity in the intestine of nematodes.

\section{Discussion}

In C. elegans, miRNAs have been shown to participate in the regulation of metabolic processes, cell development and lifespan determination ${ }^{31,32}$. Increasing evidence suggests that some miRNAs play critical roles in response to the toxicity of nanomaterials ${ }^{20,30}$. For example, the signaling cascade of BLI-1-let-7-HBL-1/LIN-41 is required in regulating GO-PEG toxicity. Moreover, GO-exposed mir-244 and mir-235 mutations induced the susceptibility to GO toxicity in decreasing lifespan. In contrast, GO-exposed mir-247/797, mir-73/74 and mir-231 mutations induced the resistance to GO toxicity in increasing lifespan. In this study, using prolonged exposure from L1 for $96 \mathrm{~h}$, we found that mir-235 mutant nematodes were sensitive to $100 \mu \mathrm{g} / \mathrm{L} \mathrm{GO}$ exposure in inducing intestinal ROS production and in decreasing locomotion behavior (Fig. S2), suggesting that prolonged exposure to GO at environmentally relevant concentration induces a mir-235-mediated response in nematodes.

miRNA, depending on the specific tissue in which it is expressed, has important functions in various biology process, such as development, longevity and toxicity of nanomaterials ${ }^{20,29,31}$. In C. elegans, miRNA expression profiling can be determined according to the miRNA driving GFP expression ${ }^{44}$. As a result, mir-235 expresses in intestine, epidermis, and neurons ${ }^{31}$. Previous studies also showed that mir-235 expresses in the hypodermic, which non-autonomously regulate $\mathrm{P}$ and $\mathrm{M}$ blast cells ${ }^{31}$. Here, we found that the mir-235 rescue in the intestine significantly inhibits the susceptibility of the mir-235 mutant to GO toxicity (Fig. 1), while the rescue of mir-235 
A
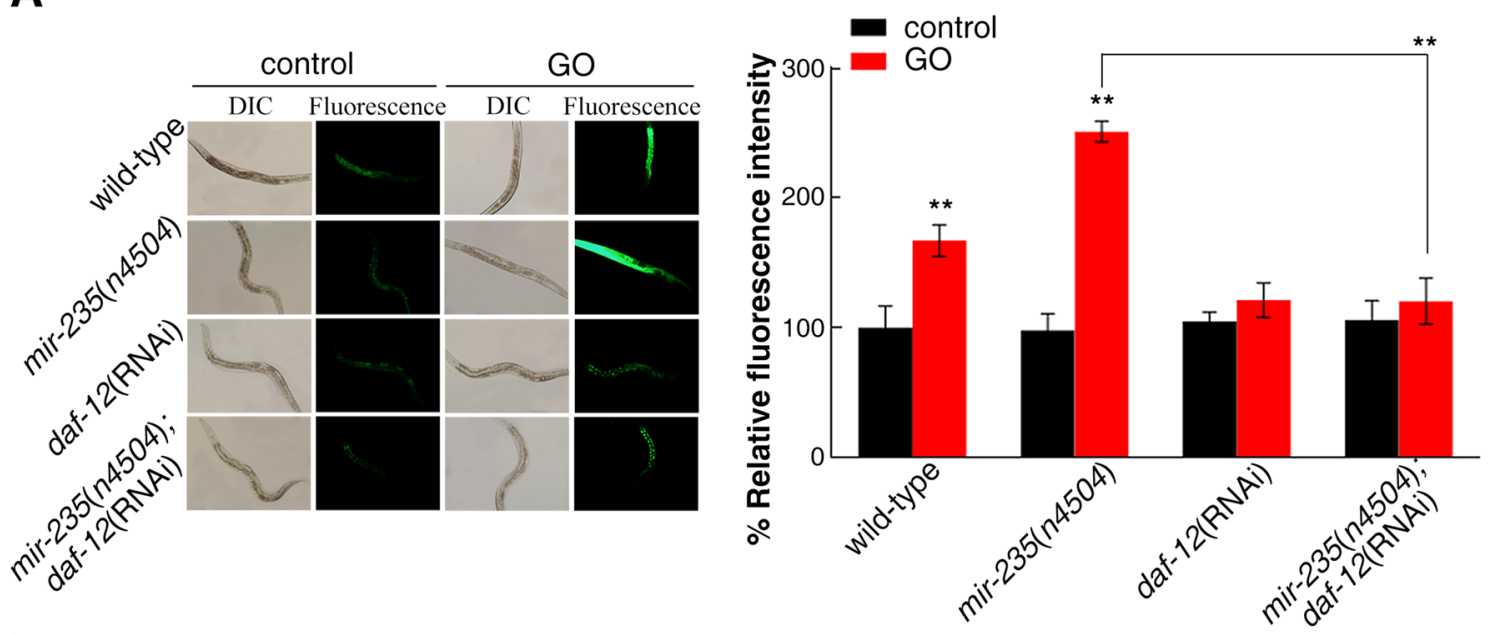

B
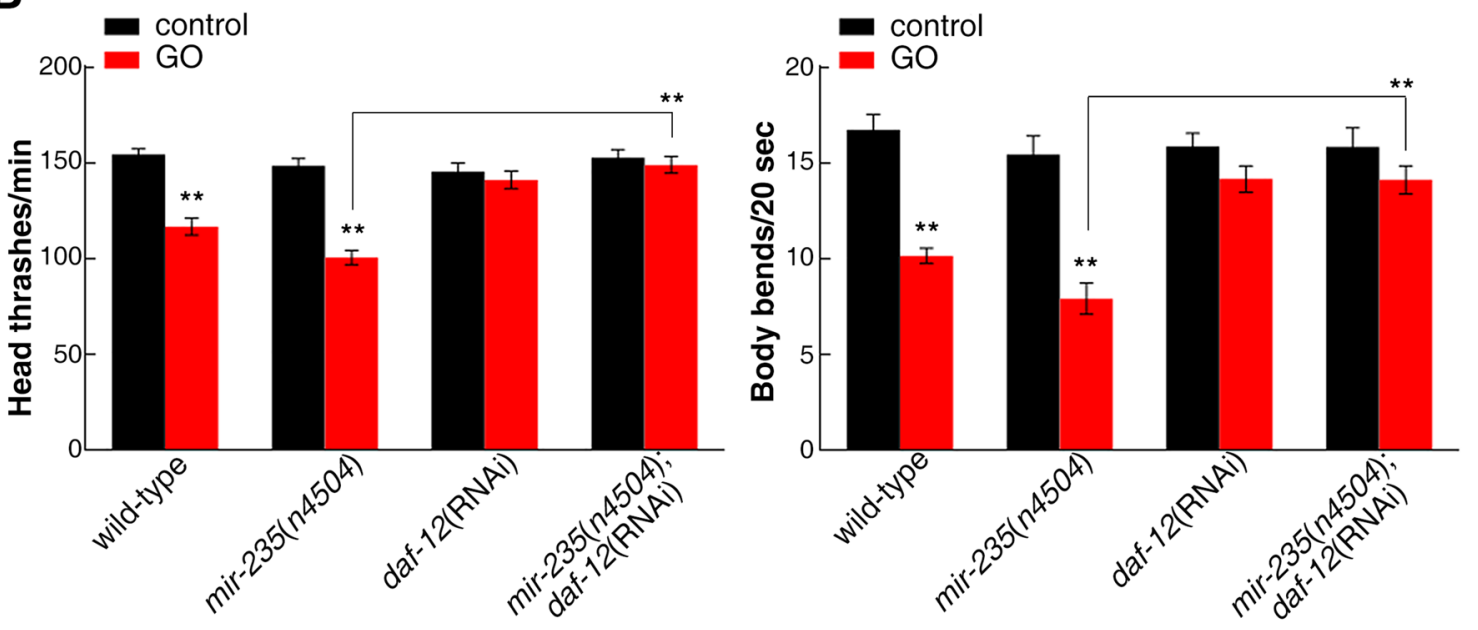

Figure 5. Genetic interactions between mir-235 and daf-12 in regulating GO toxicity. (A) Genetic interactions between mir-235 and daf-12 in regulating GO toxicity in decreasing ROS production. (B) Genetic interaction between mir-235 and daf-12 in regulating GO toxicity in increasing locomotion behavior in nematodes. Bars represent means \pm SD. ${ }^{* *} p<0.01$. Thirty nematodes were examined per treatment, and the experiment was repeated three times independently.

expression in the epidermis and neurons showed a phenotype similar to that of mir-235 mutant to GO toxicity (Fig. 1). Therefore, mir-235 acted specifically in the intestine to regulate GO toxicity.

miRNAs can target hundreds of transcripts to regulate diverse biological pathways and processes. miRNAs degrade the target mRNA or inhibit the translation of target mRNA by complementing with the $3^{\prime}$ UTR of the target mRNA $^{45}$. Studies have shown that $n h r-91$ is a targeted gene of mir-235 that involved in the regulation of development ${ }^{31}$. Besides, Hedgehog-related genes grl-5 and grl-7 are targets of mir- 235 that contribute to reactivation of quiescent neuroblasts ${ }^{46}$. Moreover, a variety of miRNAs have been found to bind target genes to regulate the toxicity of nanomaterials in C. elegans. For example, mir-231 has been proven to regulate GO toxicity via targeting smk-1 gene in C. elegans ${ }^{19}$. mab-3 serves as a target for intestinal mir-35 in regulating the response to MWCNTs $^{29}$. In this study, we found that the expression of daf-12 in the intestine of mir-235 mutant, after GO exposure, was significantly increased compared with that in the intestine of wild-type N2 (Fig. 2). Both mir-235 and daf-12 play a role in regulating GO toxicity in the intestine (Fig. 1 and Fig. $3 \mathrm{C}$ ). We have also proved that mir235 can be combined with intestinal daf-12 3' UTR (Fig. 4). Moreover, RNAi knockdown of daf-12 can reverse the susceptibility phenotype of the mir-235 mutant to GO toxicity (Fig. 5A,B). All together, our results support that daf-12 may be a direct target gene of mir-235 in the intestine in response to GO toxicity.

daf-12 encodes a nuclear receptor that regulates the dauer diapause and developmental age in C. elegans ${ }^{38}$. For example, daf-12 can activate let-7 miRNA, thereby regulating the developmental process through downstream target $h l b-1^{47}$. daf-16 is the downstream molecular of daf- 12 and regulates the lifespan of C. elegans ${ }^{48}$. Previous studies have shown that insulin and p38 MAPK signaling pathways in the intestine are involved in the regulation of GO toxicity ${ }^{13,15}$. In addition, genome-wide microarray analysis indicated that PMK-1 and DAF-16 form parallel pathways to promote immunity in C. elegans ${ }^{49}$. In this study, the genetic interaction analysis showed 


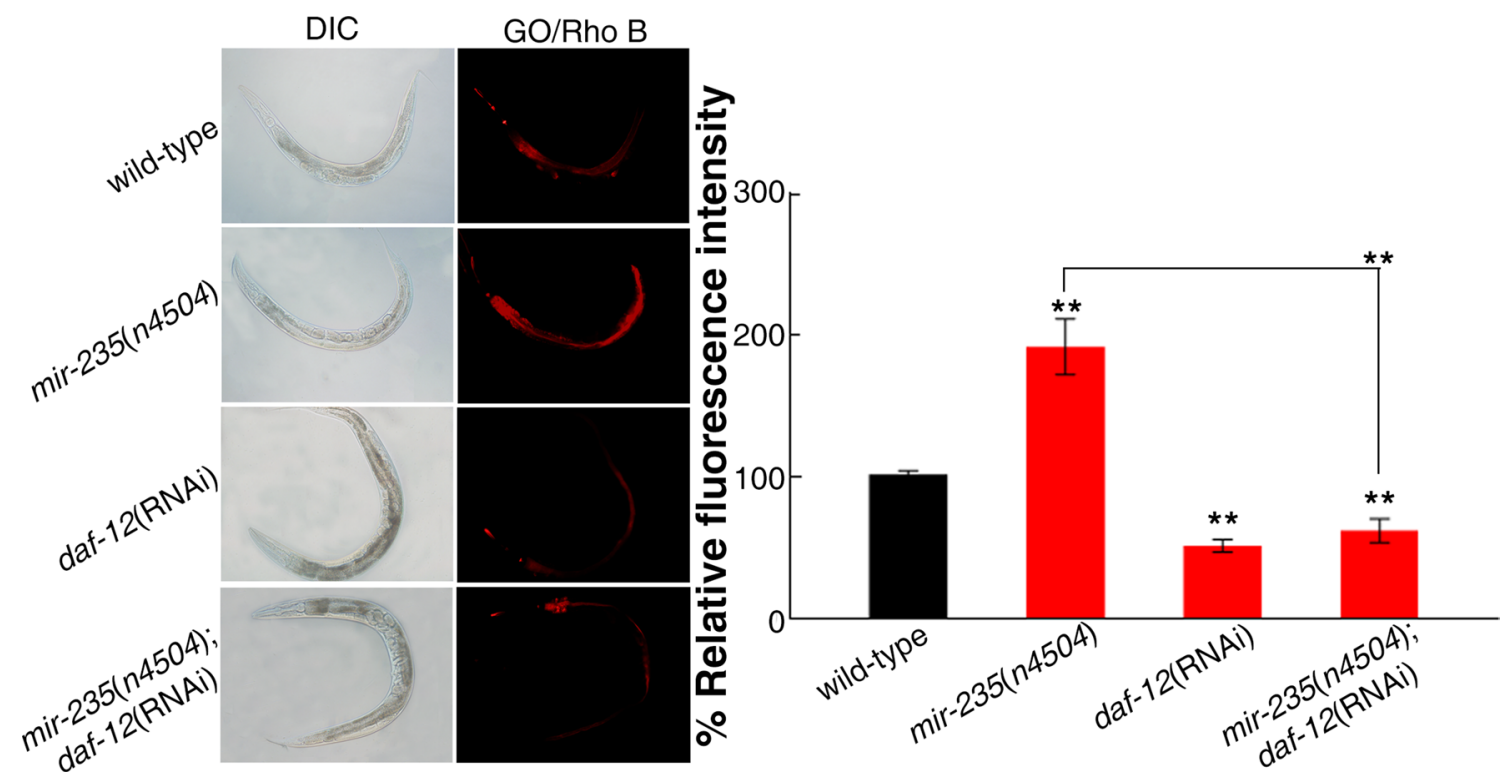

Figure 6. Distribution and translocation of GO/Rho B in nematodes. Bars represent means \pm SD. ${ }^{* *} p<0.01$. Between 25 and 30 nematodes were examined per condition, and the experiment was repeated three times independently.

that $d a f-16$ and pmk-1 also acted in parallel on the downstream of daf-12 to inhibit GO toxicity in nematodes (Fig. 8 and Fig. S5).

In conclusion, this study investigated that the potential molecular mechanisms of mir-235 medicated the response to GO exposure in C. elegans. The intestinal specificity of mir-235 in the regulation of GO toxicity was identified firstly. Importantly, we found that the mir-235 affected the toxicity of GO by influencing the function of its target gene daf-12 in the intestine. Furthermore, we demonstrated that DAF-16 in the insulin/IGF signaling pathway and PMK-1 in p38 MAPK signaling pathway acted the downstream of daf-12 and play a key role in regulating GO toxicity in parallel. Therefore, our results indicate that mir-235 mediates a protective mechanism against GO toxicity by suppressing the function of DAF-12-DAF-16 and DAF-12-PMK-1 signaling cascade in the intestine of nematodes (Fig. 9).

\section{Methods}

Reagents and preparation of GO suspensions. GO was prepared from a natural graphite powder according to the modified Hummer's method ${ }^{50}$. First, graphite $(2 \mathrm{~g})$ and sodium nitrate $(1 \mathrm{~g})$ were added in a $250 \mathrm{~mL}$ flask. Next, the concentrated $\mathrm{H}_{2} \mathrm{SO}_{4}(50 \mathrm{~mL})$ was added on ice. Then, $\mathrm{KMnO}_{4}(7 \mathrm{~g})$ was added. After the temperature of the mixture reached $35^{\circ} \mathrm{C}, 90 \mathrm{~mL}$ of deionized water was slowly dripped and stirred at $70{ }^{\circ} \mathrm{C}$ for $15 \mathrm{~min}$ to dilute the suspension. After treatment with a mixture of $7 \mathrm{~mL}$ of $30 \% \mathrm{H}_{2} \mathrm{O}_{2}$ and $55 \mathrm{~mL}$ of deionized water, the suspension was filtered to obtain a yellow-brown filter cake. The filter cake was then washed three times with $3 \% \mathrm{HCl}$, and then dried at $40{ }^{\circ} \mathrm{C}$ for $24 \mathrm{~h}$. Finally, GO would be obtained after the ultrasonication of as-made graphite oxide for $1 \mathrm{~h}$.

GO was dispersed in $\mathrm{K}_{\text {medium }}^{51}(50 \mathrm{mM} \mathrm{NaCl}, 30 \mathrm{mM} \mathrm{KCl}, 10 \mathrm{mM} \mathrm{NaOAc}$, pH 6.0) to prepare the stock solution $(1 \mathrm{mg} / \mathrm{mL})$. The stock solution was sonicated for $30 \mathrm{~min}(40 \mathrm{kHz}, 100 \mathrm{~W})$ and diluted to the appropriate concentration with $\mathrm{K}$ medium before exposure. All the other chemicals were obtained from Sigma-Aldrich (St. Louis, MO, USA).

Characterization of GO. GO was characterized by transmission electron microscopy (TEM, JEM-200CX, JEOL, Japan), atomic force microscopy (AFM, SPM-9600, Shimadzu, Japan), and Raman spectroscopy (Renishaw Invia Plus laser Raman spectrometer, Renishaw, UK) as previously described ${ }^{40}$. In addition, zeta potential was analyzed by the Nano Zetasizer using a dynamic light scattering technique (Nano ZS90, Malvern Instrument, Malvern, $\mathrm{UK})^{40}$.

Nematode strains and culture. The C. elegans strains of wild-type N2, NL2099[rrf-3(pk1426)], VP303[rde-1(ne219); kbIs7], MT17997[mir-235(n4504)], AA86[daf-12(rh61rh411)], JT204[daf-12(sa204)], CF1038[daf-16(mu86)], KU25[pmk-1(km25)] and the transgenic strain of TJ356 that carries a genome-integrated daf-16::gfp construct were obtained from Caenorhabditis Genetics Center(CGC). In addition, the transgenic strains mir-235(n4504)Ex(Pdpy-7-mir-235), mir-235(n4504)Ex(Punc-14-mir-235), mir-235(n4504)Ex(Pges-1mir-235), Ex(Pges-1-mir-235) and Ex(Pges-1-daf-12-3' UTR) used in this study were constructed in the laboratory. Nematodes were maintained on nematode growth medium (NGM) plates seeded with Escherichia coli OP50 as food source at $20^{\circ} \mathrm{C}^{52}$. 

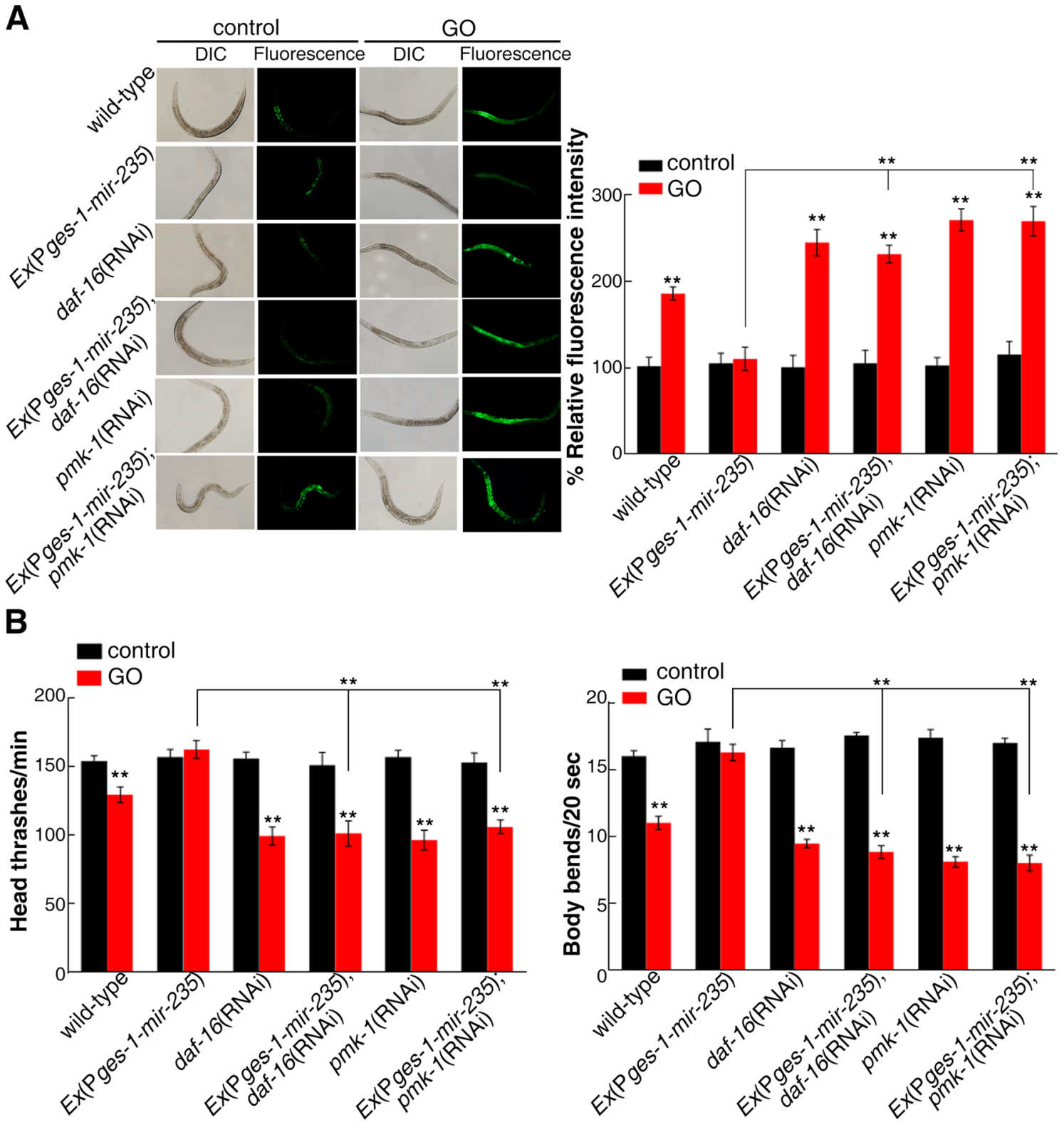

Figure 7. Genetic interactions between mir-235 and daf-16 or pmk-1 in regulating GO toxicity. (A) Genetic interactions between mir-235 and daf-16 or pmk-1 in regulating GO toxicity in inducing ROS production. (B) Genetic interaction between mir-235 and daf-16 or pmk-1 in regulating GO toxicity in decreasing locomotion behavior in nematodes. Bars represent means $\pm \mathrm{SD}$. ${ }^{\star *} p<0.01$. Thirty nematodes were examined per condition, and triplicate independent experiments were performed.

GO exposure. Age synchronous populations of L1-larvae were obtained as previously described ${ }^{53}$. GO exposure was performed from L1-larvae for $96 \mathrm{~h}$ in 12-well sterile tissue culture plates in the presence of food at $20^{\circ} \mathrm{C}^{54}$. The GO solutions were refreshed daily. The GO exposure concentration was $100 \mu \mathrm{g} / \mathrm{L}$ if not specially indicated.

Locomotion behavior. Body bends and head thrashes were used as endpoints to evaluate the locomotion behavior $^{55,56}$. After GO exposure, the nematodes were washed three times with M9 buffer, and were then transferred onto a freshly NGM plate without food to assay body bends or a freshly NGM plate without food but with $60 \mu \mathrm{L}$ M9 buffer to assay head thrashes. A body bend is calculated as a change of posterior bulb direction, and a head thrash is defined as a change for bending direction at the mid body.

Intestinal ROS production. The production of reactive oxygen species (ROS) was used to reflect the activation of oxidative stress and the functional state of the intestine ${ }^{57}$. To analyze ROS production, the examined nematodes were transferred to 12 -well sterile culture plates with $1 \mathrm{~mL}$ M9 buffer containing $1 \mu \mathrm{M}$ CM$\mathrm{H}_{2}$ DCFDA to pre-incubate for $3 \mathrm{~h}$ at $20^{\circ} \mathrm{C}$ in the dark, and then mounted on $2 \%$ agar pads for examination with a laser scanning confocal microscope (Leica, TCS SP2, Bensheim, Germany) at a $488 \mathrm{~nm}$ excitation wavelength 

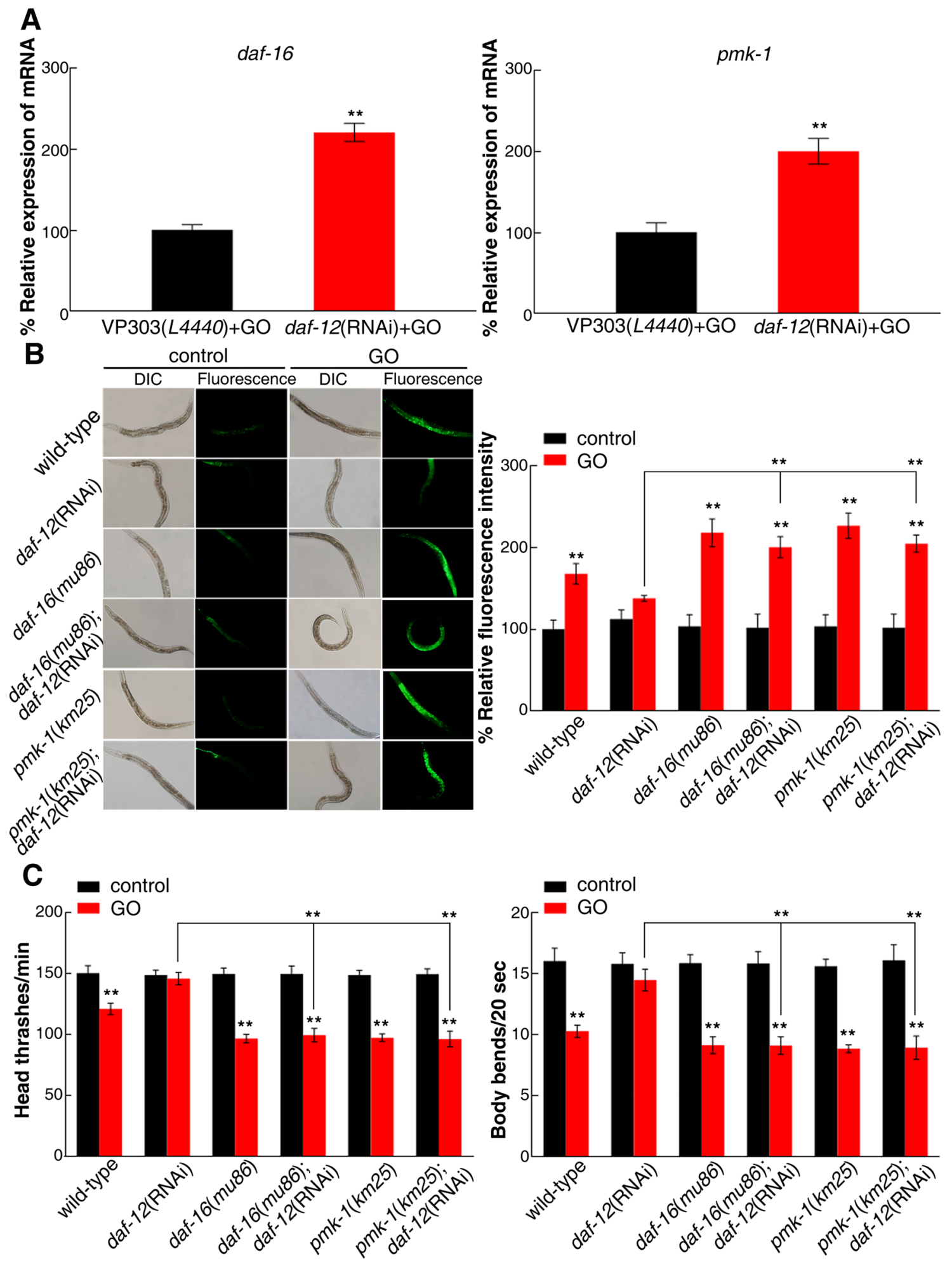

Figure 8. Genetic interactions between daf-12 and daf-16 or pmk-1 in regulating GO toxicity. (A) Effects of daf-16 and pmk-1 expression in daf-12 RNAi knockdown nematodes exposed to GO via qRT-PCR. (B) Genetic interactions between daf-12 and daf-16 or pmk-1 in regulating GO toxicity in inducing ROS production. (C) Genetic interaction between daf-12 and daf-16 or pmk-1 in regulating GO toxicity in decreasing locomotion behavior in nematodes. Bars represent means \pm SD. ${ }^{* *} p<0.01$. Thirty nematodes were examined per condition, and the experiment was repeated three times independently. 


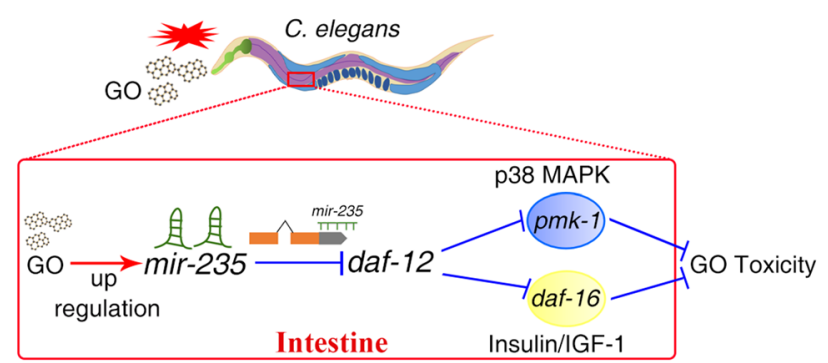

Figure 9. A diagram showing the intestinal mir-235-DAF-12-DAF-16 or mir-235-DAF-12-PMK-1 molecular signaling cascade involved in the control of GO toxicity in nematodes.

and a $510 \mathrm{~nm}$ emission filter. ROS production was semi-quantified by analyzing the fluorescent intensities, and expressed in relative fluorescent units (RFU).

Bioinformatics analysis for candidate targeted gene prediction of $\mathbf{m i r}-\mathbf{2 3 5}$. Bioinformatics software TargetScan version 6.2 (https://www.targetscan.org/worm_52/) was used to predict the possible targeted genes of mir-235. TargetScan is a tool for predicting miRNAs biological targets by finding conserved loci that match miRNA seed regions.

Dissection of nematode intestines. To the extraction of $C$. elegans intestinal RNA, we dissected the intestine of nematodes as previously described ${ }^{39}$. Nematodes were picked into $20 \mu \mathrm{L}$ M9 on a glass slide and carefully decapitated using a fine needle. Intestines were gently extracted and suspended in $50 \mu \mathrm{l}$ of M9 in an Eppendorf tube. In total 250 intestines from each treatment were collected and processed for RNA extraction.

Quantitative real-time polymerase chain reaction (qRT-PCR). Total RNA was extracted using RNeasy Mini Kit (Qiagen). Approximately 6000 nematodes were used for each treatment. Total nematode RNA $(\sim 1 \mu \mathrm{g})$ was reverse-transcribed using cDNA Synthesis kit (Bio-Rad Laboratories). Quantitative reverse transcription polymerase chain reaction (RT-PCR) was performed at the optimal annealing temperature of $52{ }^{\circ} \mathrm{C}$. The relative quantification of targeted genes in comparison to the reference $t b a-1$ gene encoding a tubulin protein was determined, and the final results were expressed as the relative expression ratio (between targeted gene and reference gene $)^{26}$. The designed primers for targeted genes and reference $t b a-1$ gene are shown in Table S3.

RNAi assay. RNAi assay was carried out by feeding nematodes with E. coli strain HT115 (DE3) expressing double-stranded RNA (dsRNA) homologous to the target gene ${ }^{58}$. E. coli HT115 (DE3) grown in LB broth containing ampicillin $(100 \mu \mathrm{g} / \mathrm{mL})$ was inoculated with ampicillin $(100 \mu \mathrm{g} / \mathrm{mL})$ and isopropyl $\beta$-D-thiogalactoside (IPTG, $5 \mathrm{mM}$ ) on NGM. L1 larvae nematodes were transferred to RNAi plates for 2 days at $20^{\circ} \mathrm{C}$ until they developed into pregnancies. The pregnant adults were transferred to fresh RNAi- expressing bacterial lawns and allowed to lay eggs to obtain the second generation RNAi nematodes. Eggs were allowed to develop into young adults for subsequent assays. Primer information for RNAi is shown in Table S4.

DNA constructs and germline transformation. To obtain vector carrying promoter sequence, promoter region for ges-1 gene specially expressed in intestine, unc-14 gene specially expressed in neurons, $d p y-7$ gene specially expressed in hypodermis, was amplified by PCR from wild-type C. elegans genomic DNA. These promoter fragments were inserted into PPD95_77 vector. mir-235 was amplified by PCR and inserted into corresponding entry vector carrying the ges-1, unc-14 or $d p y-7$ promoter sequence. Germline transformation was performed by co-injecting testing DNA $(40 \mu \mathrm{g} / \mathrm{mL})$ and marker DNA $(\mathrm{P} d o p-1:: r f p, 60 \mu \mathrm{g} / \mathrm{mL})$ into the gonad of nematodes ${ }^{59}$. Primer information for vector construction is shown in Table S5.

3'-UTR reporters and microscopy. daf-12 3' UTR (wt) was amplified by PCR from wild-type genomic DNA. daf-12 $3^{\prime}$ UTR (mu) reporter was constructed by mutating the mir-235 binding site in the daf-12 3' UTR from GUGCAAU to GAACAAU. The 3' UTR reporter containing a GFP vector driven by intestinal-specific ges1 promoter and GFP reporter under the control of the daf-12 3' UTR (wild-type or mir-235 binding site mutated) and Pges-1::mCherry unc-54 3' UTR as a control were co-injected into the gonad of nematodes as described ${ }^{59}$. Primer information for vector construction is shown in Table S5. The fluorescence intensity of the first two pairs of intestinal cells was quantified by using Image J software.

Distribution and translocation of GO. To detect the translocation and distribution of GO in nematodes, the fluorescent dye Rho B was loaded onto GO by incubating Rho B with an aqueous suspension of GO for $3 \mathrm{~h}$ as previous described ${ }^{40-42}$. Unbound Rho B was removed by dialysis against water over $72 \mathrm{~h}$. After GO/Rho B exposure, nematodes were washed with M9 buffer for three times. The distribution of fluorescence in tissues of nematodes was observed under laser scanning confocal microscope. Triplicate independent experiments were performed, and thirty nematodes were examined for per treatment. 
Statistical analysis. Results were expressed as means \pm standard deviation (SD) in this article. Figures were generated using GraphPad prism 7.00. All the data were analyzed using SPSS 12.0 software (SPSS Inc., Chicago, USA). Differences between groups were determined using analysis of variance (ANOVA). Probability levels between 0.05 and 0.01 were considered statistically significant.

Received: 23 February 2020; Accepted: 4 September 2020

Published online: 09 October 2020

\section{References}

1. Rahmanian, N., Eskandani, M., Barar, J. \& Omidi, Y. Recent trends intargeted therapy of cancer using graphene oxide-modified multifunctional nanomedicines. J. Drug Target. 25, 202-215 (2017).

2. Pang, L., Dai, C.-Q., Bi, L. \& Guo, Z.-Z. Biosafety and antibacterial ability of graphene and graphene oxide in vitro and in vivo. Nanoscale. Res. Lett. 12, 564-572 (2017).

3. Lakshmanan, R. \& Maulik, N. Graphene-based drug delivery systems in tissue engineering and nanomedicine. Can. J. Physiol. Pharmacol. 96, 869-878 (2018).

4. Pelin, M. et al. Graphene and graphene oxide induce ROS production in human HaCaT skin keratinocytes: the role of xanthine oxidase and NADH dehydrogenase. Nanoscale 10, 11820-11830 (2018).

5. Thangamuthu, M., Hsieh, K. Y., Kumar, P. V. \& Chen, G. Graphene-and graphene oxide-based nanocomposite platforms for electrochemical biosensing applications. Int. J. Mol. Sci. 20, 2975 (2019).

6. Ma, J. et al. Crucial role of lateral size for graphene oxide in activating macrophages and stimulating pro-inflammatory responses in cells and animals. ACS Nano 9, 10498-10515 (2015).

7. Zhao, Y.-L., Yang, R.-L., Rui, Q. \& Wang, D.-Y. Intestinal insulin signaling encodes two different molecular mechanisms for the shortened longevity induced by graphene oxide in Caenorhabditis elegans. Sci. Rep. 6, 24024 (2016).

8. Ayoubi, M. et al. Biochemical mechanisms of dose-dependent cytotoxicity and ROS-mediated apoptosis induced by lead sulfide/ graphene oxide quantum dots for potential bioimaging applications. Sci. Rep. 7, 1-10 (2017).

9. Feng, X.-L. et al. Graphene oxide induces p62/SQSTM-dependent apoptosis through the impairment of autophagic flux and lysosomal dysfunction in PC12 cells. Acta Biomater. 81, 278-292 (2018).

10. Park, H. H., Jung, Y. \& Lee, S. Survival assays using Caenorhabditis elegans. Mol. Cells 4, 90-99 (2017).

11. Wu, T.-S., Xu, H.-S., Liang, X. \& Tang, M. Caenorhabditis elegans as a complete model organism for biosafety assessments of nanoparticles. Chemosphere 221, 708-726 (2019).

12. Mashock, M. J. et al. Copper oxide nanoparticles impact several toxicological endpoints and cause neurodegeneration in Caenorhabditis elegans. PLoS ONE 11, e0167613 (2016).

13. Zhao, Y.-L. et al. p38 MAPK-SKN-1/Nrf signaling cascade is required for intestinal barrier against graphene oxide toxicity in Caenorhabditis elegans. Nanotoxicology 10, 1469-1479 (2016).

14. Qu, M., Li, H., Wu, Q.-L., Xia, Y.-K. \& Wang, D.-Y. Neuronal ERK signaling in response to graphene oxide in nematode Caenorhabditis elegans. Nanotoxicology 11, 520-533 (2017).

15. Ren, M.-X., Zhao, L., Lv, X. \& Wang, D.-Y. Antimicrobial proteins in the response to graphene oxide in Caenorhabditis elegans. Nanotoxicology 11, 578-590 (2017).

16. Zhi, L.-T. et al. Graphene oxide induces canonical Wnt/ $\beta$-catenin signaling-dependent toxicity in Caenorhabditis elegans. Carbon 113, 122-131 (2017).

17. Wang, D.-Y. Nanotoxicology in Caenorhabditis elegans 113-138 (Springer, Berlin, 2018).

18. Zhao, Y.-L., Jia, R.-H., Qiao, Y. \& Wang, D.-Y. Glycyrrhizic acid, active component from Glycyrrhizae radix, prevents toxicity of graphene oxide by influencing functions of microRNAs in nematode Caenorhabditis elegans. Nanomedicine 12, 735-744 (2016).

19. Yang, R.-L., Ren, M.-X., Rui, Q. \& Wang, D.-Y. A mir-231 regulated protection mechanism against the toxicity of graphene oxide in nematode Caenorhabditis elegans. Sci. Rep. 6, 32214-32214 (2016).

20. Zhao, L. et al. Dysregulation of let-7 by PEG modified graphene oxide in nematodes with deficit in epidermal barrier. Ecotoxicol. Environ. Saf. 169, 1-7 (2019).

21. Lee, R. C., Feinbaum, R. L. \& Ambros, V. T. The C. elegans heterochronic gene lin-4 encodes small Rnas with antisense complementarity to lin-14. Cell 75, 843-854 (1993).

22. Pocock, R. Invited review: decoding the microRNA response to hypoxia. Pflugers. Arch. 461, 307-315 (2011).

23. Bartel, D. P. MicroRNAs: genomics, biogenesis, mechanism, and function. Cell 116, 281-297 (2004).

24. Zendjabil, M., Favard, S., Tse, C., Abbou, O. \& Hainque, B. The microRNAs as biomarkers: what prospects?. C. R. Biol. 340, 114-131 (2017).

25. Snieckute, G. et al. mir-234 controls neuropeptide release at the Caenorhabditis elegans neuromuscular junction. Mol. Cell. Neurosci. 98, 70-81 (2019).

26. Zhao, Y.-L., Yang, J.-N. \& Wang, D.-Y. A microRNA-mediated insulin signaling pathway regulates the toxicity of multi-walled carbon nanotubes in nematode Caenorhabditis elegans. Sci. Rep. 6, 23234 (2016).

27. Zhuang, Z.-H. et al. Function of RSKS-1-AAK-2-DAF-16 signaling cascade in enhancing toxicity of multi-walled carbon nanotubes can be suppressed by mir-259 activation in Caenorhabditis elegans. Sci. Rep. 6, 32409 (2016).

28. Zhao, L., Wan, H.-X., Liu, Q.-Z. \& Wang, D.-Y. Multi-walled carbon nanotubes-induced alterations in microRNA let-7 and its targets activate a protection mechanism by conferring a developmental timing control. Part. Fibre. Toxicol. 14, 27 (2017).

29. Zhao, Y.-L., Jin, L., Wang, Y., Kong, Y. \& Wang, D.-Y. Prolonged exposure to multi-walled carbon nanotubes dysregulates intestinal mir-35 and its direct target MAB-3 in nematode Caenorhabditis elegans. Sci. Rep. 9, 12144 (2019).

30. Wu, Q.-L., Zhao, Y.-L., Zhao, G. \& Wang, D.-Y. microRNAs control of in vivo toxicity from graphene oxide in Caenorhabditis elegans. Nanomedicine 10, 1401-1410 (2014).

31. Kasuga, H., Fukuyama, M., Kitazawa, A., Kontani, K. \& Katada, T. The microRNA miR-235 couples blast-cell quiescence to the nutritional state. Nature 497, 503-506 (2013).

32. Xu, Y.-P., He, Z.-D., Song, M.-J., Zhou, Y.-F. \& Shen, Y.-D. A microRNA switch controls dietary restriction-induced longevity through Wnt signaling. EMBO Rep. 20, e46888 (2019).

33. Deusing, D. J., Beyrer, M., Fitzenberger, E. \& Wenzel, U. Carnitine protects the nematode Caenorhabditis elegans from glucoseinduced reduction of survival depending on the nuclear hormone receptor DAF-12. Biochem. Biophys. Res. Commun. 460, 747-752 (2015).

34. Wang, Z. et al. The nuclear receptor DAF-12 regulates nutrient metabolism and reproductive growth in nematodes. PLoS Genet. 11, e1005027 (2015).

35. Colella, E., Li, S. \& Roy, R. Developmental and cell cycle quiescence is mediated by the nuclear hormone receptor coregulator DIN-1S in the Caenorhabditis elegans dauer larva. Genetics 203, 1763-1776 (2016). 
36. Doudrick, K., Herckes, P. \& Westerhoff, P. Detection of carbon nanotubes in environmental matrices using programmed thermal analysis. Environ. Sci. Technol. 46, 12246-12253 (2012).

37. Gottschalk, F., Sonderer, T., Scholz, R. W. \& Nowack, B. Modeled environmental concentrations of engineered nanomaterials $\left(\mathrm{TiO}_{(2)}, \mathrm{ZnO}, \mathrm{Ag}, \mathrm{CNT}\right.$, Fullerenes) for different regions. Environ. Sci. Technol. 43, 9216-9222 (2009).

38. Antebi, A., Yeh, W., Tait, D., Hedgecock, E. M. \& Riddle, D. L. daf-12 encodes a nuclear receptor that regulates the dauer diapause and developmental age in C. elegans. Genes Dev. 14, 1512-1527 (2000).

39. Lightfoot, J. W., Chauhan, V. M., Aylott, J. W. \& Rodelsperger, C. Comparative transcriptomics of the nematode gut identifies global shifts in feeding mode and pathogen susceptibility. BMC Res. Notes 9, 142 (2016).

40. Wu, Q.-L., Zhao, Y.-L., Fang, J.-P. \& Wang, D.-Y. Immune response is required for the control of in vivo translocation and chronic toxicity of graphene oxide. Nanoscale 6, 5894-5906 (2014).

41. Zhang, R.-Y., Hummelgard, M., Lv, G. \& Olin, H. Real time monitoring of the drug release of rhodamine B on graphene oxide. Carbon 49, 1126-1132 (2011).

42. Zhang, L.-M., Xia, J.-G., Zhao, Q.-H., Liu, L.-W. \& Zhang, Z.-J. Functional graphene oxide as a nanocarrier for controlled loading and targeted delivery of mixed anticancer drugs. Small 6, 537-544 (2010).

43. Lapierre, L. R. \& Hansen, M. Lessons from C. elegans: signaling pathways for longevity. Trends Endocrinol. Metab. 23, 637-644 (2012).

44. Martinez, N. J. et al. Genome-scale spatiotemporal analysis of Caenorhabditis elegans microRNA promoter activity. Genome Res. 18, 2005-2015 (2008).

45. Ambros, V. \& Ruvkun, G. Recent molecular genetic explorations of Caenorhabditis elegans microRNAs. Genetics 209, 651-673 (2018).

46. Kume, M., Chiyoda, H., Kontani, K., Katada, T. \& Fukuyama, M. Hedgehog-related genes regulate reactivation of quiescent neural progenitors in Caenorhabditis elegans. Biochem. Biophys. Res. Commun. 520, 532-537 (2019).

47. Hammell, C. M., Karp, X. \& Ambros, V. R. A feedback circuit involving let-7-family miRNAs and DAF-12 integrates environmental signals and developmental timing in Caenorhabditis elegans. Proc. Natl. Acad. Sci. USA 106, 18668-18673 (2009).

48. Zhang, Z.-K. et al. Effects of lycium barbarum polysaccharides on health and aging of C. elegans depend on daf-12/daf-16. Oxid. Med. Cell Longev. 2019, 6379493 (2019).

49. Troemel, E. R. et al. p38 MAPK regulates expression of immune response genes and contributes to longevity in C. elegans. PLoS Genet. 2, e183 (2006).

50. Kovtyukhova, N. I. et al. Layer-by-layer assembly of ultrathin composite films from micron-sized graphite oxide sheets and polycations. Chem. Mater. 11, 771-778 (1999).

51. Wu, Q.-L. et al. Contributions of altered permeability of intestinal barrier and defecation behavior to toxicity formation from graphene oxide in nematode Caenorhabditis elegans. Nanoscale 5, 9934-9943 (2013).

52. Stiernagle, T. Maintenance of C. elegans. In WormBook, 1-11 (2006).

53. Donkin, S. \& Williams, P. L. Influence of developmental stage, salts and food presence on various end points using Caenorhabditis elegans for aquatic toxicity testing. Environ. Toxicol. Chem. 14, 2139-2147 (1995).

54. Brenner, S. The genetics of Caenorhabditis elegans. Genetics 77, 71-94 (1974).

55. Yu, C. \& Liao, V. Arsenite induces neurotoxic effects on AFD neurons via oxidative stress in Caenorhabditis elegans. Metallomics 6, 1824-1831 (2014).

56. Li, Y.-P. et al. High concentration of vitamin $\mathrm{E}$ decreases thermosensation and thermotaxis learning and the underlying mechanisms in nematode Caenorhabditis elegans. PLoS ONE 8, e71180 (2013).

57. Wu, Q.-L., Zhao, Y.-L., Li, Y.-P. \& Wang, D.-Y. Susceptible genes regulate the adverse effects of $\mathrm{TiO}_{2}-\mathrm{NPs}_{\text {at }}$ aredicted environmental relevant concentrations on nematode Caenorhabditis elegans. Nanomed. Nanotechnol. 10, 1263-1271 (2014).

58. Kamath, R. S., Martinez-Campos, M., Zipperlen, P., Fraser, A. G. \& Ahringer, J. Effectiveness of specific RNA-mediated interference through ingested double stranded RNA in C. elegans. Genome Biol. 2, 1-10 (2000).

59. Mello, C. C., Kramer, J. M., Stinchcomb, D. \& Ambros, V. R. Efficient gene transfer in C. elegans: extrachromosomal maintenance and integration of transforming sequences. EMBO J. 10, 3959-3970 (1991).

\section{Acknowledgements}

This work was supported by the grants from National Natural Science Foundation of China (21577016), and China Scholarship Council (201806095001).

\section{Author contributions}

Q.W. designed the project. T.G. and L.C. carried out the experiments. H.Z., Y.L., Y.Y. and J.L. discussed the results and reviewed the manuscript. Q.W. and T.G. wrote the manuscript.

\section{Competing interests}

The authors declare no competing interests.

\section{Additional information}

Supplementary information is available for this paper at https://doi.org/10.1038/s41598-020-73712-x.

Correspondence and requests for materials should be addressed to Q.W.

Reprints and permissions information is available at www.nature.com/reprints.

Publisher's note Springer Nature remains neutral with regard to jurisdictional claims in published maps and institutional affiliations. 
(c) (i) Open Access This article is licensed under a Creative Commons Attribution 4.0 International cc) License, which permits use, sharing, adaptation, distribution and reproduction in any medium or format, as long as you give appropriate credit to the original author(s) and the source, provide a link to the Creative Commons licence, and indicate if changes were made. The images or other third party material in this article are included in the article's Creative Commons licence, unless indicated otherwise in a credit line to the material. If material is not included in the article's Creative Commons licence and your intended use is not permitted by statutory regulation or exceeds the permitted use, you will need to obtain permission directly from the copyright holder. To view a copy of this licence, visit http://creativecommons.org/licenses/by/4.0/.

(C) The Author(s) 2020 